\title{
Review: 1 in 8 preschool children develop $\geq 1$ complication after presenting to primary care with acute cough
}

\author{
Hay $A D$, Wilson $A D$. The natural history of acute cough in children aged 0 to 4 years in primary care: a systematic \\ review. Br J Gen Pract 2002;52:401-9.
}

\begin{abstract}
QUESTION: In children 0-4 years of age presenting with the symptom of acute cough or an undifferentiated acute respiratory tract infection with cough (UARTIC) in primary care, what is the natural history of illness duration and complications?
\end{abstract}

\begin{abstract}
Data sources
Studies were identified by searching Medline (1966 to September 1998), EMBASE/Excerpta Medica (1988 to September 1998), the Cochrane Library databases, Bandolier, and the Drug and Therapeutics Bulletin using the content terms respiratory tract infection (RTI), cough, bronchitis, and chest infection; and by reviewing references of relevant articles.
\end{abstract}

Studies published in English were selected if they were cohort studies, control or placebo arms of randomised controlled trials (RCTs), or systematic reviews of RCTs, and if patients were preschool children $0-4$ years of age presenting with acute cough in primary care. For study selection, the review used the symptom of cough or UARTIC defined as a cough that failed to differentiate

\section{Study selection}

For correspondence: Dr A D Hay, Division of Primary Health Care, University of Bristol, Bristol, UK alastair.hay@bristol.ac.uk into a recognised clinical picture with known associated bacterial or viral aetiology, such as croup, whooping cough, pneumonia, or bronchiolitis. UARTIC included conditions labelled upper RTI, bronchitis, chest infection, and postnasal drip. Studies examining nonUARTIC, including pertussis and persistent wheezing, were excluded, as were those where a primary care physician was not consulted or the research was based in developing countries.

\section{Data extraction}

1 author screened $>6000$ titles online and the grey literature, scrutinised 405 of these, and excluded 384.2 authors independently assessed 21 studies for relevance and excluded 11. Each then independently extracted data on study characteristics, patient demographics, patient inclusion and exclusion criteria, recruitment and follow up rates, duration of follow up, and outcomes for the remaining 10 studies. The main outcomes were duration of illness and complications.

\section{COMMENTARY}

In this review, Hay and Wilson have tackled the difficult job of collecting data on acute cough illness in children from several studies. However, 2 limitations of the studies in the review deserve particular note: (1) the paucity of physician data on illness duration at first consultation in these studies threatens the validity of the conclusions about the duration of symptoms. If the 1 study that did collect this information is any indicator, illness duration at first visit varies widely, making calculation of total duration questionable. ${ }^{1}$ In the study by Gulbrandsen et $a l, 49 \%$ and $24 \%$ of children were unwell at $<4$ days and at $>2$ weeks, respectively. ${ }^{1}$ (2) The initiator of repeat consultation (parent or physician) varied among the studies. The criteria for judging improvement, resolution, or duration of symptoms are likely to be different for each of these 2 groups.

RTIs are a major cause of morbidity in young children. Paediatricians and family physicians report that RTIs account for $>10 \%$ of their office visits. ${ }^{2}$ The cost is large in terms of child care and lost parent work time. The lack of prognostic information is a major stumbling block to the campaign to reduce antibiotic use in respiratory illnesses. The conclusions of Hay and Wilson confirm what many practitioners know about UARTIC: the best indication that treatment is needed is probably a prolonged duration of symptoms. Experienced practitioners know that some children will develop complications. In the case of chronic cough, persistence of symptoms could be caused by bronchiolar hyperreactivity, persistent postnasal drip, or secondary bacterial invasion.

This review provides the only available summary data about duration of symptoms and percentages of complications in acute cough. It assists practitioners in providing information and advice to parents and suggests that a "wait and see" approach may be a sensible firstline treatment.

Stephanie Wright, RN, CFNP, CPNP, PhD Georgetown University School of Nursing and Health Studies Washington, DC, USA

1 Gulbrandsen P, Fugelli P, Kvarstein G, et al. The duration of acute respiratory tract infections in children. Scand JPrim Health Care 1989;7:219-23.

2 Davy T, Dick PT, Munk P. Self-reported prescribing for antibiotics for children with undifferentiated acute respiratory tract infections with cough. Pediatr Infect Dis J 1998;17:457-62.

\section{Main results}

2 cohort studies and 8 RCTs met the selection criteria. $65 \%$ of all children (where age data were given) were 0-4 years of age. At 1 week after consultation, the percent of children recovered or improved varied: $86 \%$, $75 \%$, and $8 \%$ in 3 studies where illness duration was measured by parent completed symptom diaries, and $51 \%$ in 1 study where illness duration was measured by physician assessment as "recovered"; about half were still coughing ( $49 \%$ in 1 study) and/or had nasal discharge (55\% in 2 studies). At 2 weeks, $\leq 24 \%$ of children in 1 study had not "recovered" or were worse (physician assessment), and $\leq 66 \%$ still had symptoms in another study (illness duration measured by parent completed diaries). Meta-analysis of 6 studies $(\mathrm{n}=1777)$ estimated that $12 \%$ (95\% CI $11 \%$ to $14 \%)$ of children had $\geq 1$ complication, such as rash, painful ears, diarrhoea, vomiting, or progression to bronchitis/pneumonia within 2 weeks of presentation.

\section{Conclusions}

In children 0-4 years of age who presented with acute cough in primary care, $24 \%$ of children in 1 study were judged by the physician to not have recovered or to be worse 2 weeks after the initial consultation. Metaanalysis of 6 studies shows that $12 \%$ develop $\geq 1$ complication, such as rash, painful ears, or bronchitis/ pneumonia. 